Supporting Information

for

\title{
Crucial Role of Fluorescence in the Solid-State Thermochromism of Salicylideneanilines
}

by Jun Harada, Toshikatsu Fujiwara, Keiichiro Ogawa,

Department of Basic Science, Graduate School of Arts and Sciences, The University of Tokyo, Komaba, Meguro-ku, Tokyo 153-8902, Japan ogawa@ramie.c.u-tokyo.ac.jp, harada@ramie.c.u-tokyo.ac.jp 


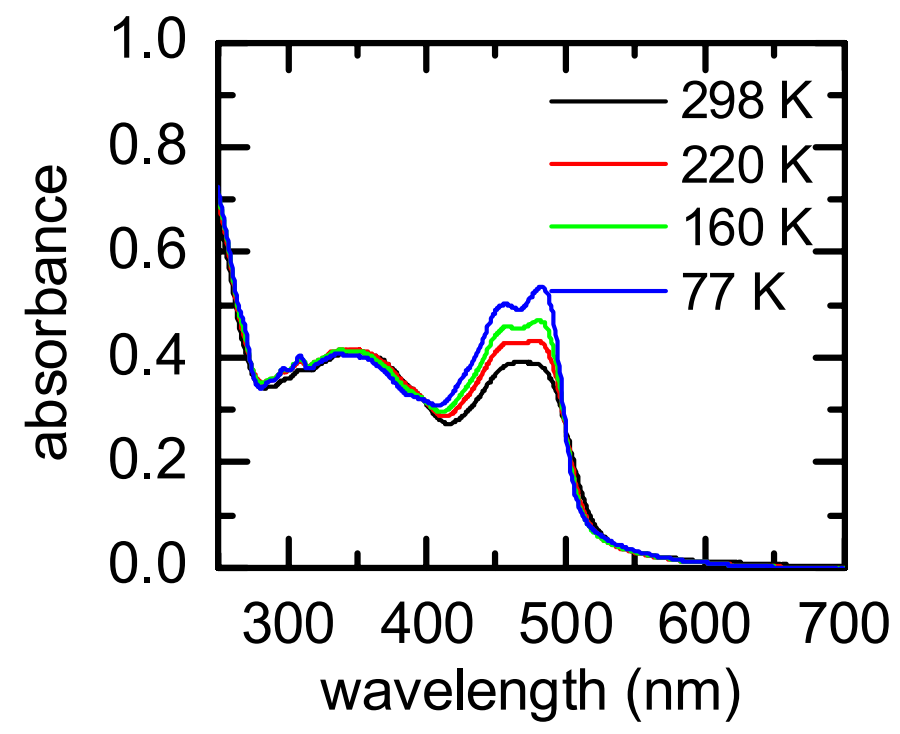

Figure S1. UV-vis absorption spectra of the crystalline film of

4-hydroxy- $N$-(5-choloro-2-hydroxybenzylidene)-aniline (3) at different temperatures.

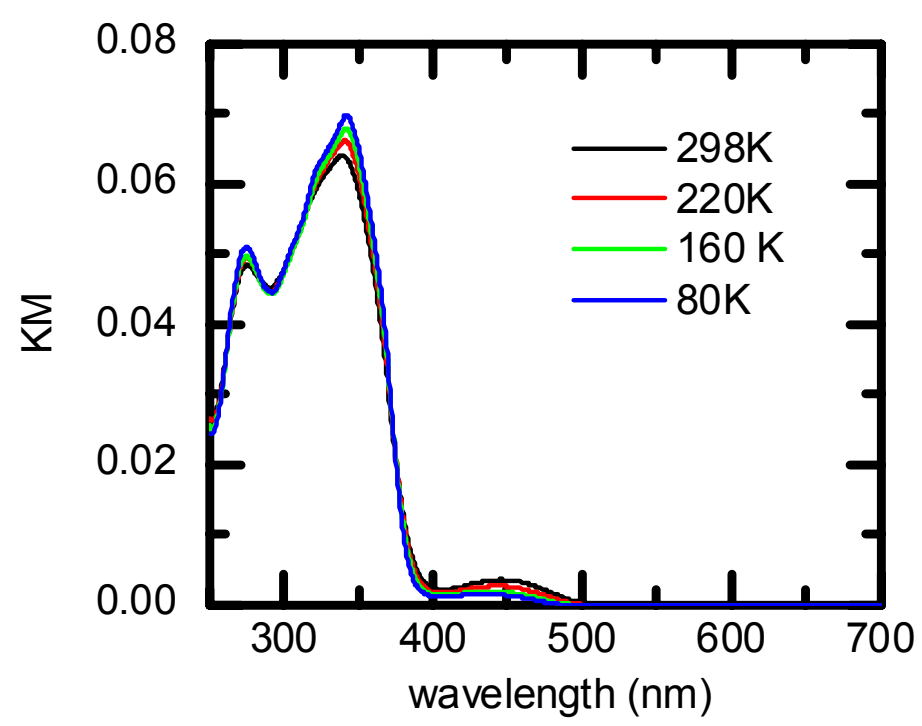

Figure S2. The Kubelka-Munk spectra of 4-methyl- $N$-(2-hydroxybenzylideneaniline) (4) in $\mathrm{NaCl}(0.1 \mathrm{wt} \%)$ at different temperatures. 


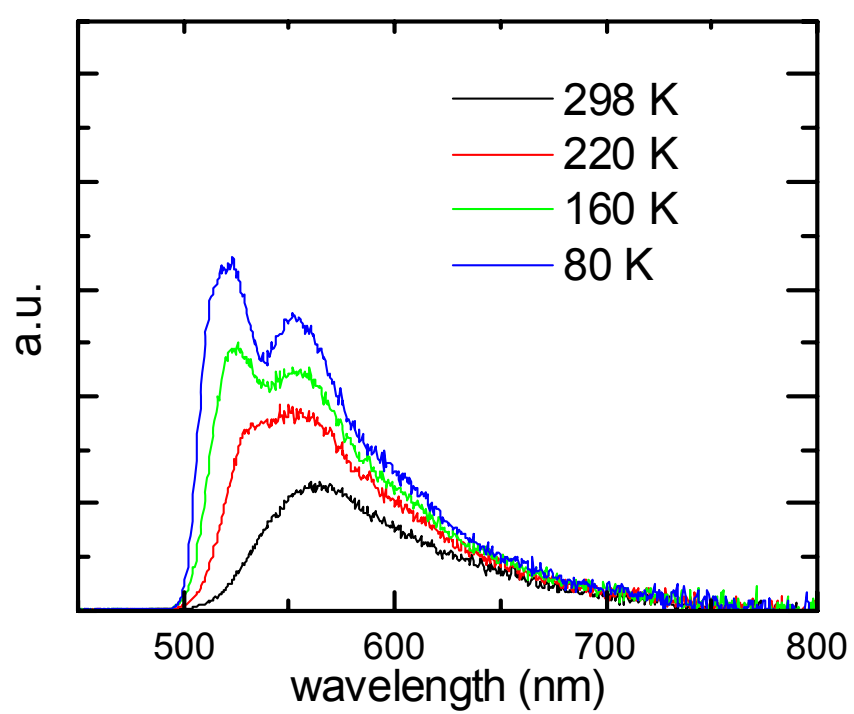

Figure S3. Fluorescence spectra of the powder of 4-methyl- $N$-(2-hydroxybenzylideneaniline) (4) at different temperatures (excitation wavelength is $420 \mathrm{~nm}$ ).

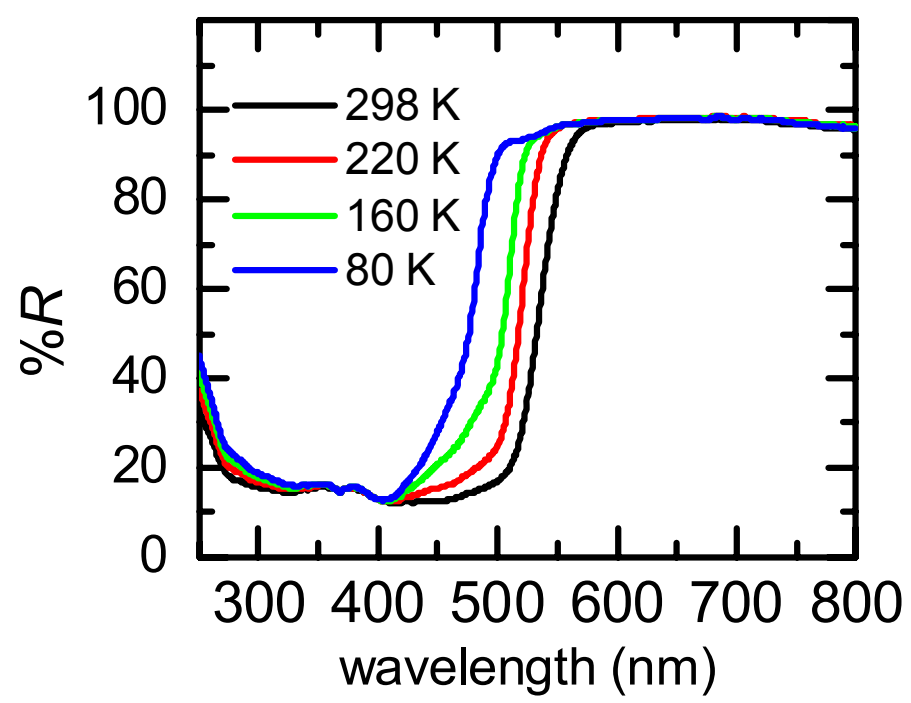

Figure S4. Diffuse reflectance spectra of 4-methyl- $N$-(2-hydroxybenzylideneaniline) (4) at different temperatures. 